\title{
MATHEMATICAL
}

PROCEEDINGS

(formerly Proceedings)

of the

Cambridge Philosophical Society

\author{
VOLUME 116
}

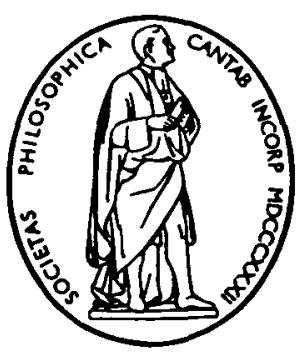


Published by the Press Syndicate of the University of Cambridge

The Pitt Building, Trumpington Street, Cambridge CB2 1RP 40 West 20th Street, New York, NY 10011-4211, USA

10 Stamford Road, Oakleigh, Melbourne 3166, Australia

(C) Cambridge University Press 1994

Printed in Great Britain by the University Press, Cambridge 


\section{INDEX FOR VOLUME 116}

PAGE

Abercrombie, A. G. Subgroups and subrings of profinite rings . . . . . . . . $\quad .209$

Aravinda, C. S. Bounded geodesics and Hausdorff dimension _ . . . . . . . . . 505

Ashwin, P. \& Stork, P. Permissible symmetries of coupled cell networks . . . . . 27

Baker, R. C. \& Brüdern, J. On sums of two squarefull numbers . . . . . . . . 1

Barnett, C., Goldstein, S. \& Wilde, I. Quantum stochastic integration and quantum stochastic differential equations

Basile, A., Bukhvalov, A. V. \& Yakubson, M. Y. The generalized Yosida-Hewitt theorem 527

Baxter, L. A. \& Li, L. Renewal theory in a random environment . . . . . $\quad .179$

Benammar, M. \& Evans, W. D. On the Friedrichs extension of semi-bounded difference operators.

Bertin, J. \& Vanhaecke, P. The even master system and generalized Kummer surfaces . 131

Brunetti, M. A family of $2(p-1)$-sparse cohomology theories and some actions on $h^{*}\left(B C_{p^{n}}\right) 223$

Brüdern, J. \& Baker, R. C. On sums of two squarefull numbers. . . . . . . . $\quad$. 1

Budak, T., Isik, N. \& Pym, J. S. Subsemigroups of Stone-Čech compactifications . . . 99

Bukhvalov, A. V., Yakubson, M. Y. \& Basile, A. The generalized Yosida-Hewitt theorem 527

Byott, N. P. Corrigendum: Some self-dual local rings of integers not free over their

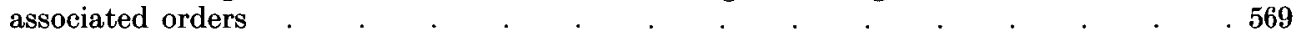

Carlson, J. F. \& Robinson, G. R. Varieties and modules with vanishing cohomology .245

Dales, H. G. \& Jarchow, H. Continuity of homomorphisms and derivations from algebras

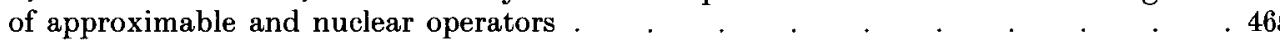

Darko, P. W. The $L^{2}$-ə-problem on manifolds with piecewise strictly non-smooth pseudoconvex boundaries. . . . . . . . . . . . . . . . . . . . . . 147

Dietz, J. Stable splittings of classifying spaces of metacyclic 2-groups. . . . $\quad$. 285

Donkin, S. On Schur algebras and related algebras III: integral representations $\quad . \quad$. 37

Echterhoff, S. Duality of induction and restriction for abelian twisted covariant systems . 301

El-Mabhouh, A. A construction of a compact right topological semigroup. . . . . 317

Evans, W. D. \& Benammar, M. On the Friedrichs extension of semi-bounded difference operators.

Fiedler, T. Triple points of unknotting discs and the Arf invariant of knots

Gambaudo, J.-M., Guaschi, J. \& Hall, T. Period-multiplying cascades for diffeomorphisms of the disc

Girela, D., Lorente, M. \& Sarrión, M. D. Embedding derivatives of weighted Hardy spaces into Lebesgue spaces

Goldstein, S., Wilde, I. \& Barnett, C. Quantum stochastic integration and quantum stochastic differential equations

Grant, K. \& Van-Brunt, B. Hyperbolic Weingarten surfaces _ . . . . . . . . 489

Greim, P., Jamison, J. E. \& Kamińska, A. Almost transitivity of some function spaces . 475

Guaschi, J., Hall, T. \& Gambaudo, J.-M. Period-multiplying cascades for diffeomorphisms of the dise

Hall, T., Gambaudo, J.-M. \& Guaschi, J. Period-multiplying cascades for diffeomorphisms of the disc

Hartley, B. \& Zalesskii, A. E. The ideal lattices of the complex group rings of finitary special and general linear groups over finite fields.

Huebschmann, J. Holonomies of Yang-Mills connections for bundles on a surface with disconnected structure group . 
Işik, N., Pym, J. S. \& Budak, T. Subsemigroups of Stone-Čech compactifications . $\quad$. 99

Jamison, J. E., Kamińska, A. \& Greim, P. Almost transitivity of some function spaces . 475

Jarchow, H. \& Dales, H. G. Continuity of homomorphisms and derivations from algebras of approximable and nuclear operators . _ . . . . . . . . . . . . . . . 465

Jones, K. N. \& Reid, A. W. Non-simple geodesics in hyperbolic-3-manifolds . . 339

Kamińska, A., Greim, P. \& Jamison, J. E. Almost transitivity of some function spaces . 475

Kaniuth, E. \& Markfort, A. Locally compact groups whose conjugation representations satisfy a Kazhdan type property or have countable support. . . . . . . . 79

Katz, D. Complexes acyclic up to integral closure _ . . . . . . . . . . . . . . 401

Lau, A. T., Milnes, P. \& Pym, J. S. Compactifications of locally compact groups and quotients.

Le Maohua. A note on the diophantine equation $\left(x^{m}-1\right) /(x-1)=y^{n} . \quad . \quad . \quad . \quad .38$

Li, L. \& Baxter, L. A. Renewal theory in a random environment . . . . . . . 179

Lorente, M., Sarrión, M. D. \& Girela, D. Embedding derivatives of weighted Hardy spaces into Lebesgue spaces . . . . . . . . . . . . . . . . 151

Lyubashenko, V. Existence of R matrix for a quantized Kac-Moody algebra . . . 193

Markfort, A. \& Kaniuth, E. Locally compact groups whose conjugation representations satisfy a Kazhdan type property or have countable support.

Mason, A. W. \& Odoni, R. W. K. Free quotients of subgroups of the Bianchi groups whose kernels contain many elementary matrices

Milnes, P., Pym, J.S. \& Lau, A. T. Compactifications of locally compact groups and

Odoni, R. W. K. \& Mason, A. W. Free quotients of subgroups of the Bianchi groups whose kernels contain many elementary matrices

Pan, $\mathrm{Y} . \mathrm{H}^{1}$ boundedness of oscillatory singular integrals with degenerate phase functions .353

Pan, Y. Convolution estimates for some degenerate curves . . . . . . . . . . . . . . 143

Peres, Y. The self-affine carpets of McMullen and Bedford have infinite Hausdorff measure 513

Pym, J. S., Budak, T. \& Işik, N. Subsemigroups of Stone-Čech complifications . . $\quad$. 99

Pym, J.S., Lau, A. T. \& Milnes, P. Compactifications of locally compact groups and quotients.

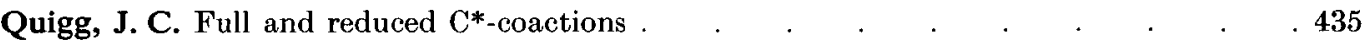

Reid, A. W. \& Jones, K. N. Non-simple geodesies in hyperbolic 3-manifolds . . . 339

Robinson, G. R. \& Carlson, J. F. Varieties and modules with vanishing cohomology . 245

Ruškuc, N. On the rank of completely 0 -simple semigroups _ . . . . . . . . . $\quad 325$

Sarrión, M. D., Girela, D. \& Lorente, M. Embedding derivatives of weighted Hardy spaces into Lebesque spaces ..$\quad$. . . . . . . . . . . . . . 151

Skowroński, A. Minimal representation-infinite artin algebras _ . . . . . . . . . . . 229

Smart, N. P. $S$-integral point on elliptic curves . . . . . . . . . . . . . . . . 391

Stafford, J. T. \& Zhang, J. J. Examples in non-commutative projective geometry . . . 415

Stork, P. \& Ashwin, P. Permissible symmetries of coupled cell networks . _ . . . . . 27

Stratmann, B. Diophantine approximation in Keinian groups _ . . . . . . . . . $\quad 57$

Suleiman, I. A. I. \& Wilson, R. A. The 2-modular characters of Conway's group $\mathrm{Co}_{2} \quad .275$

Tadie. On the bifurcation of steady vortex rings from a Green function . . . . . 555

Teragaito, M. Roll-spun knots (Corrigenda). . . . . . . . . . . . . . . . . . 191

Van-Brunt, B. \& Grant, K. Hyperbolic Weingarten surfaces $\quad$. . . . . . . . . . . 489

Vanhaecke, P. \& Bertin, J. The even master system and generalized Kummer surfaces . 131

Wilde, I., Barnett, C. \& Goldstein, S. Quantum stochastic integration and quantum stochastic differential equations . . . . . . . . . . . . . . . . . 535

Wilson, R. A. \& Suleiman, I. A. I. The 2-modular characters of Conway's group $\mathrm{Co}_{2} \quad .275$ 
Yakubson, M. Y., Basile, A. \& Bukhvalov, A. V. The generalized Yosida-Hewitt theorem 527 Zalesskii, A. E. \& Hartley, B. The ideal lattices of the complex group rings of finitary special and general linear groups over finite fields .. \begin{tabular}{lllll}
\hline &. &. & & \\
\hline & & 7
\end{tabular}

Zhang, J. J. \& Stafford, J. T. Examples in non-commutative projective geometry . 415 


\section{THE PREPARATION OF MANUSCRIPTS}

\section{The attention of authors is particularly directed to the following requests.}

1. Papers should be typed, double-spaced, on one side of white paper (of which A4, 210 by $297 \mathrm{~mm}$, is a suitable size). The pages must be numbered. Margins of $30 \mathrm{~mm}$ should be left at the side, top and bottom of each page. Two clear copies should be sent.

A cover page should give the title, the author's name and institution, with the address to which mail should be sent.

The title, while brief, must be informative (e.g. A new proof of the prime-number theorem, whereas Some applications of a theorem of $G$. H. Hardy would be useless).

The first paragraph or two should form a summary of the main theme of the paper, providing an abstract intelligible to mathematicians.

For a typescript to be accepted for publication, it must accord with the standard requirements of publishers, and be presented in a form in which the author's intentions regarding symbols etc. are clear to a printer (who is not a mathematician).

The following notes are intended to help the author in preparing the typescript. New authors may well enlist the help of senior colleagues, both as to the substance of their work and the details of setting it out correctly and attractively.

\section{Notation}

Notation should be chosen carefully so that mathematical operations are expressed with all possible neatness, to lighten the task of the compositor and to reduce the chance of error.

For instance $n_{k}$ ( $n$ sub $k$ ) is common usage, but avoid if possible using $c \operatorname{sub} n$ sub $k$. Fractions are generally best expressed by a solidus. Complicated exponentials like

should be shown in this and no other way.

$$
\exp \left\{z^{2} \sin \theta /\left(1+y^{2}\right)\right\}
$$

In the manuscript, italics, small capitals and capitals are specified by single, double and triple underlinings. Bold faced type is shown by wavy underlining; wavy will be printed wavy.

It helps if displayed equations or statements which will be quoted later are numbered in order on the right of their line. They can then be referred to by, for example, 'from (7)'.

The author must enable the printer (if necessary by pencilled notes in the margin) to distinguish between similar symbols such as $0, O, o, 0,0 ; x, \mathrm{X}, \times ; \phi, \Phi, \varnothing ; \mathrm{I}, \mathrm{I} ; \varepsilon, \in ; \kappa, k$.

Greek letters can be denoted by $\mathrm{Gk}$ in the margin.

If an author wishes to mark the end of the proof of a theorem, the sign I may be used.

Footnotes should be avoided.

\section{Diagrams}

It is extremely helpful if diagrams are drawn in Indian ink on white card, faintly blue or green-lined graph paper, or tracing cloth or paper. Symbols, legends and captions should be given on a transparent overlay. Each text figure must be numbered as Figure 1, Figure $2, \ldots$ and its intended position clearly indicated in the manuseript:

\section{Figure 1 here}

The author's name in pencil must be on all separate sheets of diagrams.

A figure is expensive to reproduce and should be included only when the subject matter demands it, or when it greatly clarifies the exposition.

The Society recognizes that some authors do not have the facilities for producing drawings of a sufficiently high standard to be reproduced directly and it is therefore willing to have such diagrams redrawn, provided that they are clear.

4. Tables

Tables should be numbered (above the table) and set out on separate sheets. Indicate the position of each in the text as for figures:

Table 3 here

\section{References}

References should be collected at the end of the paper numbered in alphabetical order of the authors' names. Titles of journals should be abbreviated as in Mathematical Reviews. The following examples show the preferred style for references to a paper in a journal, a paper in a proceedings volume, a book and an unpublished dissertation:

[1] J. F. Adass. On the non-existence of elements of Hopf invariant one. Ann. of Math. (2) 72 (1960), 20-104.

[2] M. P. Fotrman and D. S. Scotr. Sheaves and logic. In Applications of Sheaves, Lecture Notes in Math. vol. 753 (Springer-Verlag, 1979), pp. 302-401.

[3] P. T. Johsstone. Stone Spaces. Cambridge Studies in Advanced Math. no. 3 (Cambridge University Press, 1982).

[4] F. W. Lawtere. Functorial semanties of algebraic theories. Ph.D. thesis. Columbia University (1963). 


\section{Mathematical Proceedings of the Cambridge Philosophical Society}

MPCPCO 116 (Pt 3) 385-576 (1994) 0305-0041 November 1994

\section{CONTENTS}

LE MАонш. A note on the diophantine equation $\left(x^{m-1}\right) /(x-1)=y^{n}$

SMART, N. P. S-integral points on elliptic curves . . . . . . . . . . . . 391

Katz, D. Complexes acyelic up to integral closure . . . . . . . . . . . . 401

StAFFoRd, J. T. \& ZHANG, J.J. Examples in non-commutative projective geometry . . . 4I5

Qureg, J.C. Full and reduced $C^{*}$-coactions . . . . . . . . . . . 435

LaU, A. T., Munes, P. \& PYM, J. S. Compaetifications of locally eompact groups and quotients . 451

DALES, H. G. \& JARCHOW, H. Continuity of homomorphisms and derivations from algebras of approximable and nuclear operators

465

Grem, P., Jamson, J. E. \& Kamísska, A. Almost transitivity of some function spaces . 475

VAN-Brunt, B. \& GRANT, K. Hyperbolic Weingarten surfaces . . . . . . . 489

ARAvivd A, C. S. Bounded geodesies and Hausdorff dimension . . . . . . . 505

PEREs, Y. The self-affine carpets of McMullen and Bedford have infinite Hausdorff measure . 513

Basme, A., Bukhyalov, A. V.\& YakuBson, M. Y. The generalized Yosida-Hewitt theorem 527

BARnetr, C., Goldsters, S. \& WHDE, I. Quantum stochastie integration and quantum stochastic differential equations

TaDIE. On the bifurcation of steady vortex rings from a Green function . . . . . .

Byotr. N. P. Corrigendum: Some self-dual local rings of integers not free over their associated orders

Procendings of Mentings 